Aus den Universitäts-Augenliniken in Breslan (Direktor: Geheimrat Prof. Dr. W. Uhth off) und Tubingen (Direktor: Prof. Dr. G. ₹. Schleich)].

\title{
Arbeiten aus dem Gebiete der Pathologie des Zentralgefässystems der Netzhaut (Gefässystem und Auge).
}

IV. Mitteilung ${ }^{1}$.

\section{Arterielle Anastomosenbildung in der Netzhaut.}

\section{Yon}

\author{
Privatdozent Dr. Clemens Harms, \\ Tübingen.
}

Mit Taf. IX, Fig. 1, und 3 Figuren im Text.

\begin{abstract}
"Wirkliche Neubildung von Anastomosen, wie sie in Pagenstechers (2) Fall von Sehnerventrauma mit Zerreissung der Zentralgetässe sehr bald auf der Papille sichtbar wurde, scheint nach Embolie nicht vorgekommen zu sein, wenn man nicht das Konvolut äusserst feiner Gefässe, das Hock(1) einmal 32 Tage nach der Erblindung im äusseren Teile der Papille vorfand, das aber mit den Netzhautgefässen nicht in wahrnehmbarem Zusammenhange stand, als einen freilich verfehlten Anlauf hierzu betrachten will", sagt Fischer (4, S. 114) in seiner grossen Monographie (1891): "Über die Embolie der Arteria centralis retinae", während Gonin(13) die ausserordentliche Seltenheit des Vorkommens wirklicher arterieller Anastomosenbildung in der Netzhaut in seiner im Jahre 1905 publizierten Arbeit über dieses Thema folgendermassen zum Ausdruck bringt: "Les rares exemples d'anastomoses entre artères rétiniennes, qui ont été publiés, appartiennent à des états si complexes qu'ils ne sauraient être donnés comme de véritables exceptions à une règle d'ailleurs générale."
\end{abstract}

\section{Eigene Beobachtungen.}

Ich bin in der angenehmen Lage, drei neue Beobachtungen dieser seltenen Fälle im folgenden mitteilen zu können. Der erste der-

1) IIr. Mitteilung siehe v. Graefe's Areh. f. Ophth. Bd, LXXXVI, 3. S.514. 
selben, dessen Publikation Herr Prof. v. Schleich mir freundlichst erlaubte, wurde bereits im Jahre 1902 in der Tübinger UniversitätsAugenklinik von uns beobachtet.

\section{Fall I1). (Hierzu Textfigur 1.)}

47jähriger Mann mit Herzleiden nach Endocarditis bei Gelenkrhenmatismus. Ältere Obliteration der rechten Arteria temporalis inferior auf eine Strecke von 1 P.D. (ungefähr 2 P.D. von der Papille entfernt) mit zwei anastomotisehen Verbindungen (aben und unten) des zentralen und peripheren Arterienstückes und $z$ wei anastomotischen Verbindungen $z$ wischen dem peripberen Arterienstücke und einer kleineren, weiter temporal verlaufenden Arterie. Zugleieh frischer Verschluss (mit zentraler Trübung der Netzhaut und rotem Fleck in der Macula lutea) einer aus der Art. temp. sup. entspringenden makularen Arterie, deren peripheres Ende mit einer kleineren unteren makularen Arterie in anastomotischer Verbindung steht.

Melchior W., 47 Jahre alter Tagelöhner aus A., kommt am 15 . VIII. 1902 (Journ.-Nr. 4087) in die Tübinger Universitäts-Augenklinik wegen einer angeblich erst seit zwei Wochen bestehenden hochgradigen Sehstörung seines rechten Auges. Mit dem linken Auge hat er, wie er angibt, von Jugend auf sehlecht gesehen, während das Selurermögen des rechten Anges immer gut gewesen sein soll, so dass er z. B. während seiner militärisehen Dienstzeit beim Schiessen nicht behindert war. Am 1. VIII. ds. Js. machte er abends einen Spaziergang, nachdem er vorher zu Bett gelegen hatte; nach etwa 10 Minuten trat plötzlich eine Sehstörung des rechten Auges ein, so dass er auf diesem Auge nur noch einen Schein hatte. Seither ist keine Anderung des Zustandes eingetreten. Von irgendwelchen früheren Versehlechterungen des Sehvermögens auf dem rechten Auge ist dem Patienten nichts bekannt. Seit sechs Jahren ist er herzleidend infolge Gelenkrheumatismus und seit März ds. Js. mit Pausen arbeitsunfähig.

Der Arzt des Patienten (Angenarzt Dr. Rieth in Aalen), der ihn der Klinik überwies, teilt mit, dass der Patient schon seit Jahren wegen Herzleidens nach Endocarditis bei Gelenkrheumatismus in seiner Behandlung stehe, und dass das linke Auge infolge hochgradiger Myopie mit atrophischen Veränderungen in der Macula schwachsichtig sei. Die jetzige Sehstörung anf dem rechten Auge, deretwegen der Patient gleich nach ihrem Auftreten bei ihm in Behandlung getreten war, habe er mit Bettruhe, Schwitzen und Jodkalinm, jedoch ohne greifbaren Erfolg behandelt.

Befund vom 15. VIII. 1902:

Links $\Delta=$ Finger in $1 \mathrm{~m}$; mit $-22,0 \mathrm{D} S=\overline{y_{20}} ;$ Jäger Nr. 1 in $4 \mathrm{~cm}$. Gesichtsfeld von unten und innen sehr eingeschränkt (bei grober Prüfung mit grösseren Gegenständen). Bei äusserlich normalen Verhältnissen

1) Dieser Fall wurde in der unter meiner Anleitung angefertigten Dissertation von. Paul Kober (19, siehe Tabelle VII, Nr. 56 und Tabelle VIII, Nr. 29) statistisch mit verwertet. 
findet sich ophthalmosk opisch ein grosses, ringförmiges Staphylom von $21 / 2$ P. D. Breite nach der temporalen Seite. Aussen unten ist ein atrophischer Herd von Papillengrösse, ausserdem besteht alte Chorioiditis ad maculam. Im ganzen ist der Fundus selrr pigmentarm.

Rechts $S=$ Handbewegungen. Äusserlich normal, die Pupille ist von gleicher Weite wie links, reagiert aber nur sehr Jangsam und selr wenig

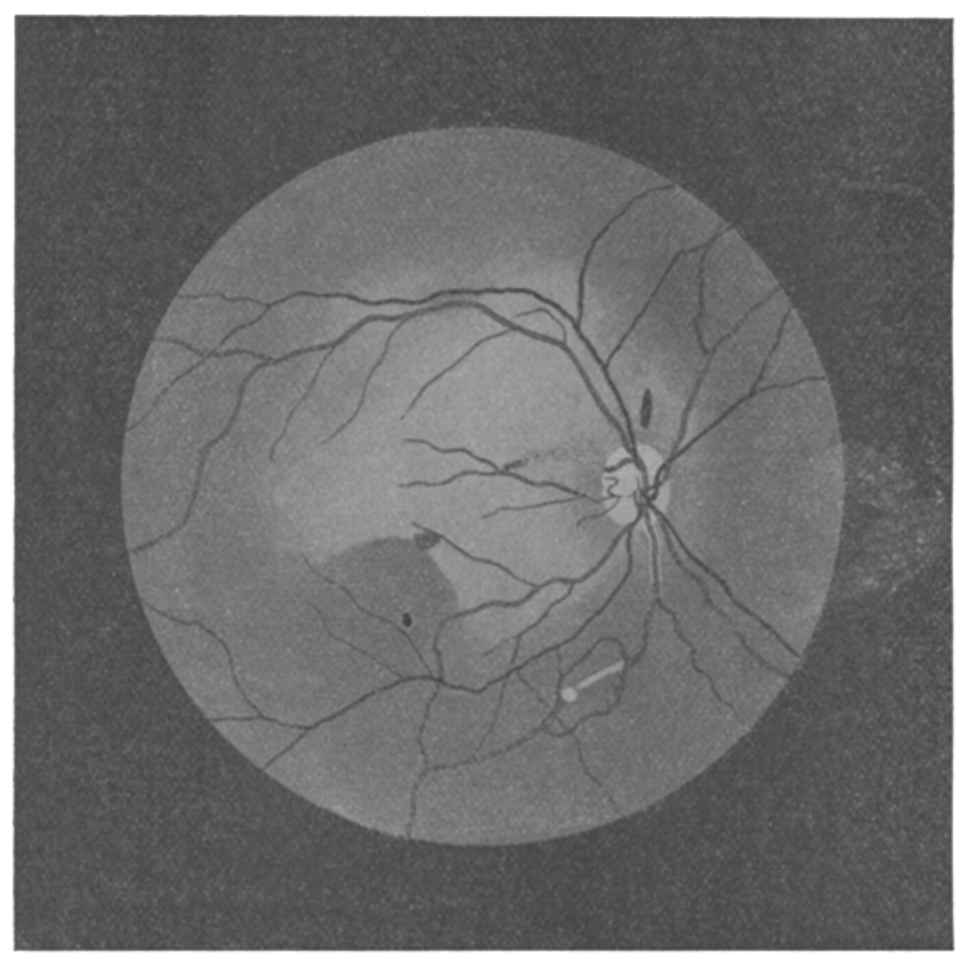

Rechtes Auge. Aufrechtes Bild.

Fig. 1 (nach einer $z$. T. farbigen Skizze von Prof. Grunert in Bremen, fruher in Tübingen) zeigt den Hintergrundsbefund des Falles I vom 15. VIII 1902 (2 Wochen nach der plotzlich eingetretenen hochgradigen Sehstörung). Rechts $S=$ Handbewegungen.

ausgiebig auf Lichteinfall, konsensuell dagegen gut. Ophthalmoskopisch (siehe Fig. 1) sind die Medien klar. Die Papille ist von senkrecht ovaler Form und in ihrer Farbe sehr blass. Die auf ihr sichtbaren Gefässe (Venen sowohl wie Arterien) sind stark verengt. Die Papille ist umgeben von einer hellgrauen Retinaltrübung, die nach unten, innen und oben innen (i. aufr. B.) sehr gering ist und hier nor eine schmale (etwa papillenbreite) und dann rasch abklingende Zone darstellt. Nach oben und besonders nach anssen ist dagegen die Trubung sehr intensiv, ähnlich wie bei Ablatio retinae, nur dass die Netzhaut hier anliegt, und die vorhandenen Gefässe deutlich rot erscheinen. Nach der Macula zu nimot die Trubung an Inten- 
sität zu und hört mit ziemlich scharfem Rande an der Macula auf. Letztere markiert sich dabei als ein dunkler, braunroter Fleck, der in einer stumpfwinkligen Einziehung der Begrenzungslinie der Trübung liegt. Diese Begrenzungslinie hat eine leicht wellige Form. Nach oben von der Papille sieht man zwischen den Gefässen eine strichförmige, vertikal verlaufende Blutung von ungefähr ${ }^{1 / 2}$ P.D. Länge. Ein gegen die Macula ziehendes Gefäss (Arterie) hört am Papillenrande plötzlich scharf abgeschnitten auf, um in einiger Entfernung von der Papille, etwas vor der Anastomosierung mit einer andern von der Papille herziehenden Arterie, wieder zu beginnen. Das ausgefallene Stück wird noch durch eine feine, bisweilen unterbrochene rote Linie angedeutet. Die Arteria temporalis inferior ist beim Austritt aus der Papille auf eine Strecke von ungefähr 1 P.D. stark eingescheidet und sehr dünn; etwa 2 P.D. unterhalb der Papille fehlt in derselben ein Stück von 1 P.D. Länge und ist ersetzt durch ein weissliches Band, das am peripheren Ende mit einer knöpfchenartigen Anschwellung endet. Um diese Unterbrechung herum verlaufen oben und unten zwei bogenförmige Anastomosen, welche das distale Ende des zentralen Stückes mit dem proximalen Ende des peripheren Stückes der unterbrochenen Arterie verbinden, und von denen die untere korkzieherartig geschlängelt ist. Ausserdem besteben zwei Anastomosen des peripheren Stuckes der un terbrochenen Arterie miteiner kleineren, weiter temporal unter der Macula verlaufenden Arterie. Fast sämtliche Gefässe, insbesondere auch die Venen, beginnen an ihrem Ursprunge auf der Papille sehr dünn, um dann in einiger Entfernung rom Optikus, oft ohne allmählichen Übergang, an Kaliber zuzunehmen. Ord. Bettruhe, Eserin und Tet. Strophanti.

Am 16. VIII. 1902 wurde die Vorderkammer des rechten Auges durch Lanzenschnitt von oben her punktiert, am 18. VIII. war die Operationswunde wieder verklebt und das Auge reizlos.

Am 21. VIII. 1902 war die Wunde fest verheilt, das Auge äusserlich normal und reizlos mit Ausnahme einer geringen Trübung der Cornea (Verbandkeratitis?). Rechts $S=$ Handbewegungen (ohne sichere Angabe der Richtung). Pupillenreaktion bei direkter Belichtung nur angedeutet, konsensuell prompt. Im Gesichtsfelde (Prüfung mit grossen Gegenständen) wird nur bei Annäherung des Gegenstandes von aussen her dio Bewegung erkannt, und zwar erst in geringer Entfernung vom Fixierpunkte. Bei der Prüfung im Dunkelzimmer mit Kerze wird Lichtschein gleichfalls nur bei Bewegung von aussen her in der Nähe des Fixationspunktes erkannt. Bei Anwendung des Hohlspiegels ist Lichtschein überall vorhanden, Projektion jedoch nur geradeaus und nach aussen. - Links mit - 20,0 D $S=\overline{5} / 15$ Jager Nr. 1.

Am 22. VIII. 1902 ist die Keratitis rechts wieder abgeheilt. Ophthalmoskopisch sieht man nach aussen am Papillenrande eine frische Blutung.

Am 23. VIII. 1902 werden rechts Handbewegungen in $1 \mathrm{~m}$ Entfernung sicher erkannt, dagegen werden Finger in der Nähe unsicher und nur excentrisch gezählt. Das Gesichtsfeld (bei Prüfung mit der Hand).

7. Graefé's Arehiv für Ophthalmologio. LXXXVH. 2. 
scheint in seinen peripheren Grenzen nach aussen, aussen oben und aussen unten ziemlich normal zu sein, die Prüfung mit Licht im Dunkelzimmer ergibt dasselbe Resultat. Ophthalmoskopisch sind beide Blutungen am Papillenrande in Resorption begriffen, in der Maculagegend finden sich feinste weisse Stippchen, sonst keine Änderung gegen friiher.

Patient wird am 24. VIII. 1902 mit Brille (R. plan, L. $-18,0$ D) und Brief an seinen Arzt entlassen und hat sich nicht wieder gezeigt.

Nach Mitteilung der Ortsbehörde vom 2.XI. 1912 ist der Patient am 14. IV. 1903 an "Tuberkulose" gestorben.

Die streckenweise Obliteration der Arteria temp. inf., die, obwohl der Patient nichts davon gemerkt hatte, scheinbar schon länger bestand, hat wohl zweifellos den Anlass zur Ausbildung der anastomotischen Verbindungen zwischen den beiden Stücken der Arterie und zwischen dem peripheren Stücke und einer unter der Macula verlaufenden kleineren temporalen Arterie gegeben. Da man jedoch die Zeit des Eintritts der Obliteration der Arterie nicht kennt, so fehlt jedes Urteil über den Zeitraum, der zur Ausbildung der Anastomosen zur Verfügung stand. Dagegen scheint sich die Anastomose zwischen den beiden makularen Arterien erst im Anschlusse an den plötzlichen Verschluss der einen (oberen), also im Laufe von ungefähr zwei Wochen vor der ersten Untersuchung in der Klinik ausgebildet zu haben. Bei so kurzer Zeit der Entwicklung einer Anastomose muss man daran denken, dass die Verbindung der beiden Gefässe durch Kapillaren schon vorgebildet war, die Anastomose also nur eine Erweiterung schon vorhandener Bahnen darstellt. Bei der anastomotischen Verbindung zweier Stiicke eines und desselben, auf eine Strecke unterbrochenen Gefässes, wie sie an der Art. temp. inf. stattgefunden hatte, ist man aber wohl berechtigt, eine wirkliche Neubildung dieser arteriellen Gefässe, welche die Verbindung herstellen, anzunehmen. Diese wird zu ihrer Entwicklung wohl auch längere Zeit gebraucht haben.

Als eine besondere und bisher wohl kaum beobachtete Eigentümlichkeit des Falles ist noch der Umstand zu betrachten, dass zwei isolierte Astverschlüsse der Zentralarterie (der Art. temp, inf. und eines makularen Astes der Art. temp. sup.) mit einem grossen Zeitabstande nacheinander erfolgten, obwohl ja aus dem Befunde und der Anamnese nicht mit Sicherheit zu ersehen ist, ob der Verschluss der Art. temp. inf. plötzlich und unter dem Bilde der sog. Embolie erfolgt war. Aber nach Analogie anderer länger beobachteter Fälle von plötzlich eintretendem Astverschluss, bei denen es auch zu einer 
streckenweisen Obliteration des befallenen Gefässastes kam, ist dieser Hergang nach dem Befunde durchaus nicht auszuschliessen.

Dass der Verschluss der beiden Gefässäste hauptsächlich auf lokalsklerotischer Basis erfolgte, geht zur Genüge aus der perivaskulären weissen Einscheidung der Arteria temporalis inferior in ihrem Anfangsteile hervor, wenn auch das infolge der Endocarditis arthritica aufgetretene "Herzleiden" bei der thrombotischen Entstehung des Verschlusses eine begünstigende Rolle gespielt haben mag. Gegen eine embolische Entstehung der beiden Verschlüsse spricht jedenfalls ausser dem Lokalbefund auch noch die Wiederholung des Prozesses an demselben Auge nach einem relativ grossen Zeitabraume, die bei Annahme einer "Embolie" als ein ganz besonders grosser Zufall anzusehen wäre.

Den zweiten Fall dieser Art, dessen Mitteilung mir von Herrn Geheimrat Uhth of f gütigst gestattet wurde, habe ich 1903 während meines Aufenthalts in Breslau zu sehen Gelegenheit gehabt.

\section{Fall II. (Hierzu Textfigur 2 und 3.)}

25 jährige Frau mit Insuffizienz und Stenose der Mitralis nach Endocarditis bei Gelenkrheumatismus im 13. Lebensjahre. Plotzlicher Verschluss der rechten Arteria nasalis inferior a af und unterhalb der Papille, nach $21_{2}$ Monaten neugebildete Anastomose $z$ wischen dem noch durchgängigen peripheren Stücke derin ihrem Anfangsteile unterbrochenen Arteria nasalis inferior und dem Anfangsteile der Arteria temporalis inferior auf der $P a p i l l e$, nach $6{ }^{1} / 2$ Monaten eine $z$ weite anastomotische Verbindung $z$ wischen dem noch durchgängigen peripheren Stücke eines an seinem Ursprunge auf eine Strecke hochgradig verengten nasalen Astes der Arteria nasalis inferior mit dem Anfangsteile der Vena nasalis superior auf der Papille.

Frau Elisabeth D., 25 Jahre alt, aus B., kommt am 27. I. 1903 (Journ.-Nr. 4238) in die Breslauer Univ.-Augenklinik mit der Angabe, dass sich vor 14 Tagen das linke Auge ganz plötzlich versehlechtert habe; dabei empfand sie, so eine Art Sehwindelanfall". Das rechte Auge habe immer sehon schlechter gesehen und habe in der Jugend geschielt. Im 5. Lebensjahre wurde eine Schieloperation vorgenommen (Tenotomie des rechten Rectus internus?). Im 13. Lebensjahre hatte sie Gelenkrheumatismus, seitdem Herzfehler. Patientin ist seit $\% / 4$ Jahren verheiratet, bisher keine Schwangerschaft.

Befund rom 27. I. 1903:

Links mit $-0,5 D S={ }^{6} / 6$. Accommodation normal. Ophthalmoskopisch besteht ein partieller Verschluss des unteren Arterienastes mit 
Trübung der unteren Hälfte der Retina, die nach der Peripherie langsam abnimmt.

Rechts mit $-0,5 D S={ }^{6} / 18 ; S n \quad 0,5$ in $15 \mathrm{~cm}$ mühsam. Kein Schielen, keine Insuffizienz der Muskeln. - Urin wegen Menses nicht zu untersuchen.

Allgemeinzustand nach der Diagnose des Hausarztes (Dr. Carl Becker in Breslau): Insuffizienz und Stenose der Mitralis.

Im Gesiehtsfelde (am 29. I. 1903 aufgenommen) findet sich ein

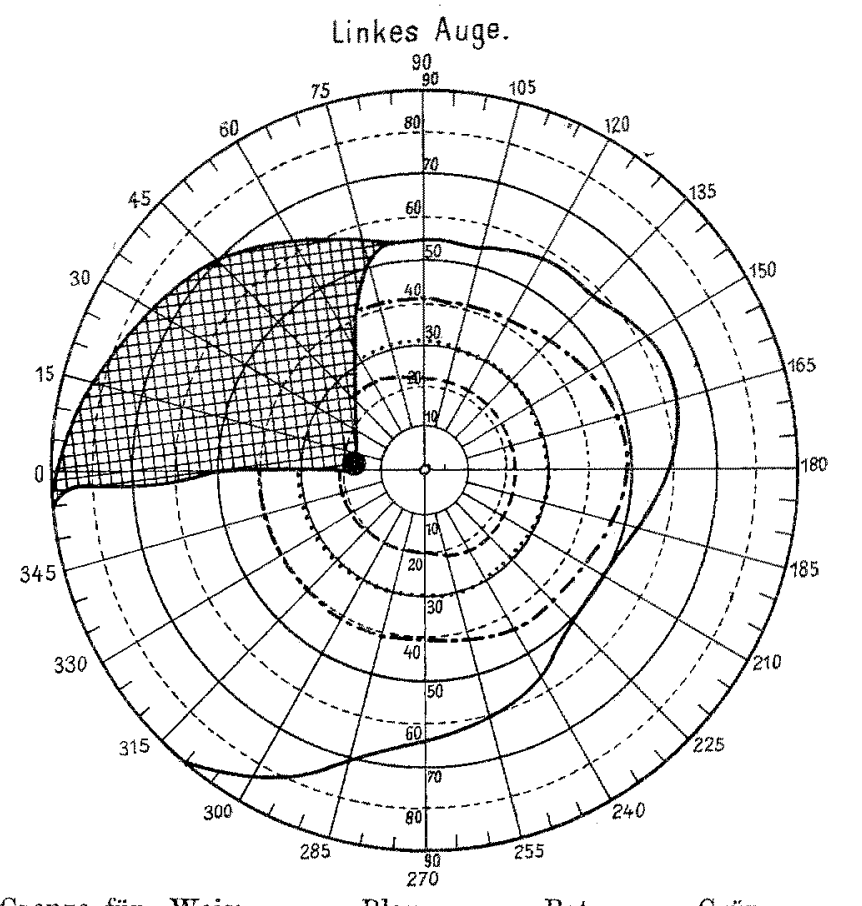

Grenze für: Weiss ; Blau

absoluter Defelst für Weiss und alle Farben.

Fig. 2. Gesichtsfeld des Falles II rom 29. 1. 1903. Links $S=5 / 8$.

absoluter Ausfall des oberen änsseren Quadranten, dessen spitze im blinden Fleek liegt (siehe Fig. 2).

Am 31. I. 1903. Links $V=6 / 8$. Ophthalmoskopiseh sieht man uber der verstopften Arterie ein viereckiges glänzendes Ding (auf der Arterienwand oder spindelförmige Auftreibung derselben?)

Am 16. Il. 1903. Links $V=6 / 8$. Ophthalmoskopisch ist das verstopfte Gefäss auf der Papille bis zur Verschlussstelle sehr eng, aber schon 2-3 Papillendurchmesser von der Papille entfernt immer noch ganz normal. Die partielle Abblassung der Papille beginnt.

Am 28. III. 1903 fand sich nach einer von Prof. Heine angefertigten 
Skizze eine anastomotische Verbindung zwischen einer peripher von dem Verschlussstüeke gelegenen Stelle der Arteria nasalis inferior und der nach unten aussen verlaufenden Arterie, ausserdem zog rom proximalen Ende des durchgängigen Teiles eines in seinem Anfangsteile hochgradig verengten nasalen Astes der Arteria nasalis inferior ein neugebildetes, stark korkzieherartig gewundenes Gefäss nach oben bis zum unteren Papillenrande, ohne mit einem andern Gefässe in direkter Verbindung zu stehen (siehe unten).

Am 28. VII. 1903 hatte ich selbst Gelegenheit, die Patientin zu unter-

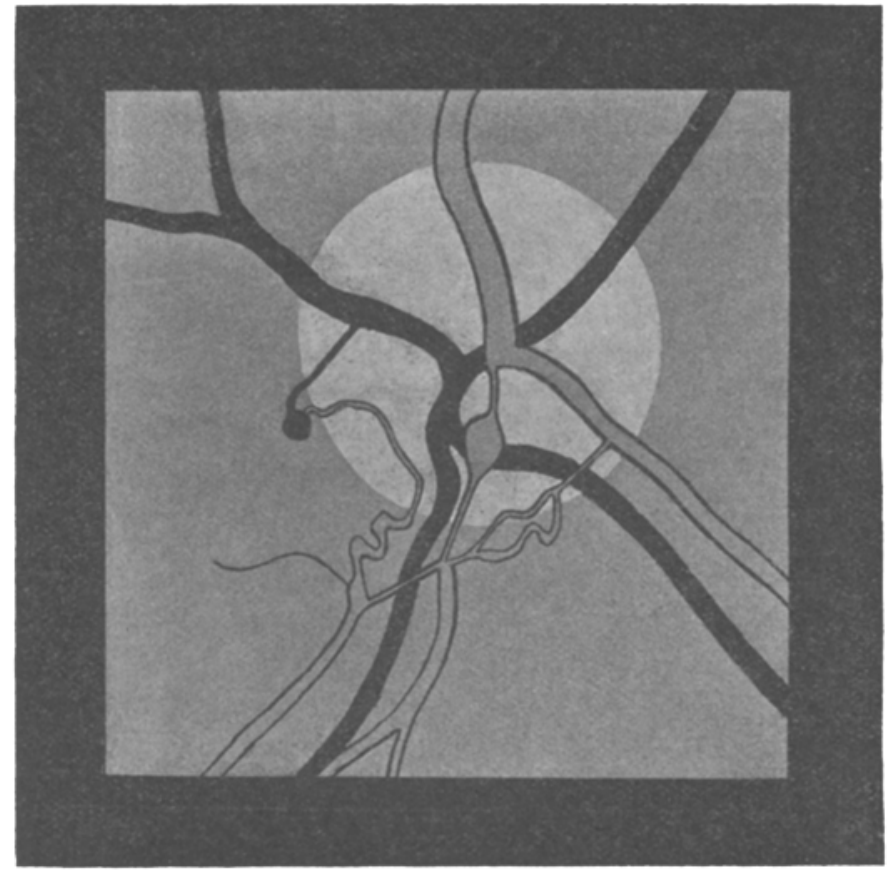

Linkes Auge. Aufrechtes Bild.

Fig. 3 [nach 2 Skizzen von Prof. Heine in Kiel (früher in Breslau) und mir] zeigt den Befund an der Papille des Falles II vom 28. VII. 1903. Links $S=5 / 8$.

suchen und eine Skizze anzufertigen. Fig. 3 ist nach den beiden von Prof. Hein $\mathrm{e}$ und mir entworfenen Skizzen der rechten Papille und Umgebung wiedergegeben. Die nach unten und etwas innen verlaufende Arterie zeigt gleich nach ihrem Abgange vom Stamme in der Gefassspforte eine hochgradige Verengerung mit feinem Blutfaden, dann erweitert sie sich spindelförmig: auf der Papille und sieht hier in dieser Partie hellglänzend aus (ohne Blutgehalt), um peripherwärts wieder in einen dünnen Strang mit feinem Blutfaden überzngehen, der sich etwas unterhalb der Papille plötzlich erweitert und nun als annähernd normales Gefäss weiter gegen die Peripherie verläuft. Genau an der Stelle des Überganges der engen in die weite Partie 
geht nach unten innen ein $A$ st $a b$, der zuerst ebeufalls als dünner Strang wit schmalem Blutfaden erscheint und als soleher die Vena nasalis inferior uberbrückt, bald nasalwärts von der Vene aber auch plötzlich weiter wird und als normales Gefäss in die Peripherie verläuft. An dieser Stelle des Überganges der engen in die weitere Partie geht ein kleiner dinner Ast fast horizontal nach innen, zugleich aber lrommt an dieser Stelle ein (nengebildeter) Arterienzweig hervor, der sich stark korkzieherartig gesehlängelt nach oben auf die Papille begibt, ein Stuick derselben (unten innen) bogenförmig überschreitet und die Papille dann nach innen wieder verlässt, um dicht neben ihrem nasalen Rande in einem rundlichen roten Fleck (Gefässknäuel?) zu enden, der dem kirschroten Maculateck bei sog. Embolie nicht unähnlich ist. Ans diesem Fleck kommt ein zweiter Ast (wohl eine Vene?) hervor, der sich gewissermassen als Fortsetzung des ersten sehräg nach oben aussen wieder auf die Papille begibt, um sich mit der Vena nasalis superior zu verbinden. Kehren wir zu dem Hauptstamme der Arteria nasalis inferior zurück, so sieht man an der Stelle des Überganges der engen in die weitere Partie, genau gegenüber dem Abgange des in seinem Anfangsteile verdünnten nasalen Astes, eine kleinere neugebildete Arterie hervorkommen, die, nach oben aussen ziehend, sich bald in zwei stark korkzieherartig geschlängelte Äste teilt, welehe auf dem unteren Papillenrande wieder zur Vereinigung gelangen. Der dünne Ast, welcher die Fortsetzung bildet, überbrückt die Vena temporalis inferior und vereinigt sich dann mit der Arteria temporalis inferior. Links $S=6 / 6-6 / 5 ;$ Nieden 1 .

Fassen wir zusammen, so besteht also eine neugebildete arterielle anastomotische Verbindung zwischen einer Stelle der Arteria nasalis inferior unterhalb der Papille (jenseits der verschlossenen oder doch hochgradig verengten Strecke auf der Papille) und dem Anfangsteile der Arteria temporalis inferior auf der Papille, sowie eine neugebildete Anastomose zwischen einer Stelle eines nasalen Astes der Arteria nasalis inferior. (ebenfalls jenseits der hochgradig verengten Anfangsstrecke) und dem Anfangsteile der Vena nasalis superior anf der Papille. Diese Anastomosen warden erstmals (am 28. III. 1903) etwa $\left.2^{1}\right|_{2}$ Monate nach Eintritt des plötzlichen Verschlusses der Arteria nasalis inferior beobachtet, während bei der Untersuchung am $16 . \mathrm{IL}$. 1903, also etwa einen Monat nach der Zirkulationsstörung, noch nichts davon zu sehen war (wenigstens steht in dem ron Heine geschriebenen Befunde nichts davon vermerkt). Sie haben offenbar den Zweck verfolgt, die Zirkulation in dem von derselben ausgeschlossenen peripheren Teile der Arteria nasalis inferior und ibres an seiner $\mathbf{A b}$ gangsstelle ebenfalls verschlossenen Astes wieder herzustellen, wodurch allerdings eine volle Wiederherstellung der Funktion nach so langer Zeit (mehr als 1 Monat) nicht zu erwarten wax. Leider liegt aber 
eine genaue Gesichtsfelduntersuchung aus der späteren Zeit nicht mehr vor, so dass nicht $\mathrm{zu}$ entscheiden ist, ob nicht doch vielleicht durch diesen natürlichen Heilungsprozess, der ja bei den arteriellen Verschlüssen zu den grössten Seltenheiten gehört, eine relative Besserung des vorher absoluten Gesichtsfelddefektes eingetreten ist, was im Hinblick auf die merkwürdige und bis jetzt einzig dastehende Beobachtung Alexanders (6) und den unten noch zu besprechenden Fall Königshöfers (11) nicht so ganz auszuschliessen wäre. Interessant ist, dass nach $2{ }^{1} / 2$ Monaten, wie aus der Heineschen Skizze hervorgeht, die von dem nasalen Aste der Arteria nasalis inferior ausgehende neugebildete Arterie erst bis zum unteren Rande der Papille ohne Verbindung mit einem andern Gefässe vorgedrungen war, während nach $61_{2}$ Monaten durch Vermittlung eines Gefässknäuels am inneren Rande der Papille die Verbindung mit der Vena nasalis superior hergestellt war.

Erst vor kurzem wurde in der Tübinger Klinik ein dritter hierhergehöriger Fall beobachtet, den ich leider selbst nicht gesehen habe.

Fall III. (Hierzu Fig. 1 auf Taf. IX.)

47 jährige Frau mit Dilatatio cordis und leisem systolischem Geräusch bei freiem Urin. Plötzliche Erblindung des rechten Auges zugleich mit Hemiplegie der rechten Hirnhemisphäre (mit Bewusstlosigkeit und Paralyse der ganzen linken Körperseite). Nach ungefähr 11 Monaten ophthalmoskopisch atrophische Papille, Obliteration aller Arterienäste auf derselben und anastonotische Verbindung aller vier erst am Rande der Papille beginnenden Hauptarterienäste durch einen Kranz von nengebildeten, stark geschlängelten Collateralen rings um den Optikus.

Die Wirtsfran Agathe F., 48 Jahre alt, aus A. 0.|A. M. wird am 28. VII. 1913 (Journ.-Nr. 6336) von der hiesigen medizinischen Klinik in die ambulante Spreehstunde der Univ.-Augenklinik geschiekt, weil sie Anfang September 1912 zugleich mit einem Schlaganfall, der eine Hemiplegie der linken Seite zur Folge hatte, eine Erblindung des rechten Auges erlitten habe, die wohl auf Embolie zurückzuführen sei. Die Patientin gab noch an, sie habe gleich in den ersten Tagen nach dem Schlaganfall ihren Arzt darauf aufmerksam gemacht, dass sie mit dem rechten Auge nichts mehr sehe. Seither sei der Zustand des erblindeten Auges unverändert geblieben, während die Lähmungserseheinungen der linken Körperhälfte zurïickgegangen seien.

Die von Herrn Professor Fleiseher über den Befund gemachten Notizen lauten folgendermassen:

Links. $\Delta=5 / 5-\left.5\right|_{4}$.

Rechts. $\Delta=$ Handbewegungen. Die Pupille reagiert nicht auf direkten 
Lichteinfall. Ophthalmoskopisch sind die brechenden Medien klar. Die Papille ist gelblich blass und nicht ganz scharf begrenzt. In der Mitte sind die Tüpfel der Lamina cribrosa zu erkennen. An den Arterien lassen sich abnorme Veränderungen konstatieren: Der Stamm der Arterie ist nicht sichtbar, dagegen sind die Arterien in der Netzhaut vorhanden und teilweise in der Nähe der Papille eingescheidet, die Venen sind stark gefüllt. Die Arterien sind von der Peripherie her bis zum Rande der Papille sichtbar, der weitere Verlauf auf der Papille fehlt, oder die Arterie ist nur ganz eng, wobei das Kaliber teilweise schwankt. Zahlreiche kleine Gefässchen, welche von der Unterbrechungsstelle der Arterien abzweigen, finden sich am Rande der Papille, wo sie sich nach stark geschlängeltem Verlaufe in diese einsenken. Offenbar handelt es sich um eine Collateralenbildung durch Erweiterung von kleinen Gefässen des Optikusrandes. Zwischen Papille und Macula findet sich eine kleine Hämorrhagie, zwei Venen sind etwas weit. Der iubrige Hintergrund ist ohne pathologisehen Befund.

Allgemeinzustand: Aus der mir freundlichst zur Verfügung gestellten Krankengeschichte der hiesigen medizinischen Klinik (Direktor Prof. Otfried Müller), wo die Patientin vom 18. VII. bis 2. VIII. 1913 aufgenommen war, entnehme ich mit freundlicher Erlaubnis des Herrn Prof. Müller noch folgendes: Nach den an amnestischen Angaben der Patientin war sie im Anschluss an den am 5. IX. 1912 erlittenen Sehlaganfall einige Tage bewusstlos, dann war die ganze linke Körperseite bewegungslos. Nach drei Wochen waren die ersten Bewegungen im linken Arm wieder möglich, dann auch im linken Bein, und allmählich wurde es immer besser, so dass sie schliesslich wieder gehen konnte. Nur seit zehn Wochen ist der Zustand ziemlich gleich geblieben. Das Gedäehtnis hat etwas gelitten, besonders für die Ereignisse der letzten Zeit, sie ist sehwer besinnlich geworden. Seit einem Jahre keine Periode mehr. - Im ganzen hat sie drei Geburten gehabt, von denen zwei normal verliefen, die eine (Nr.2) war eine Frühgeburt. Die beiden ersten Finder leben und sind gesund, das dritte ist an der (normalen) Geburt gestorben. Der Mann ist an Blutvergiftung gestorben. Patientin hat im Anschluss an ihre zweite Geburt (Frühgeburt) vor 10 bis 11 Jahren ein Herzleiden bekommen, das nach Aussage ihres Hausarztes eine Herzerweiterung war.

Bei der objektiven Untersuchung finden sich noch als Reste der vor ungefähr einem Jahre aufgetretenen Hemiparese: eine Sehwäche der linken oberen und unteren Extremität, Babinski, dauernde Dorsalflexion der Grosszehe, sehr lang dauernder Fussclonus, apoplektischer Gang mit Nachschleifen des linken Fusses, sowie leichte Facialisparese.

Am Herzen ist eine Dilatation wegen des enormen Fettpolsters schwer sicher zu stellen, aus demselben Grunde der Spitzenstoss nicht fühlbar. Die Töne sind leise, an der Mitralis leises systolisches Geräusch. Es findet sich leichte periphere Arteriosklerose. Blutdruck $=165-158 \mathrm{~mm}$ Zg. - Der Urin ist frei von Eiweiss und Zueker.

In diesem Falle kamen die uns hier interessierenden Hintergrundsveränderungen erstmals ungefähr 11 Monate nach der plötzlich 
eingetretenen Erblindung, die wohl zweifellos durch Stammverschluss der Zentralarterie erfolgte, zur Beobachtung, und es scheint, dass es sich um einen fertigen Zustand und bereits endgültig abgeschlossenen Neubildungsprozess handelt. Wie lange Zeit diese Anastomosen zu ihrer Entwicklung brauchten, lässt sich jetzt natürlich nicht mehr schätzen, aber jedenfalls waren es weniger als 11 Monate, wenn man nicht annehmen will, dass vielleicht die jetzt sichtbaren Anastomosen schon vor dem Eintritt der Erblindung vorhanden waren. Aber nach der Erfahrung meiner beiden vorhergehenden Beobachtungen wie auch der Fälle von Burnett(9), Königshöfer(11) und Coats(18) darf ich wohl vermuten, dass die Anastomosen sich erst nach der Erblindung entwickelt haben, d. h. dass der die Erblindung bedingende plötzliche Verschluss des Stammes der Zentralarterie den direkten und unmittelbaren Anreiz zur Wucherung der neugebildeten Gefässverbindungen auch in diesem Falle gab. Auch in den sehr ähnlichen Fällen von Nettleship (5) und Gonin (13), bei denen ebenfalls der neugebildete Gefässkranz nach Stammverschluss um die Papille herum lag, konnte die Anastomosenbildung nicht in ihrer succesiven Entwicklung verfolgt werden, sondern die Beobachter fanden nach 3 (Gonin), und 12 Monaten (Nettleship) den fertigen Zustand vor. A us der kurzen Beschreibung geht in beiden Fällen nicht mit Sicherheit hervor, ob die Autoren den Anfangszustand gleich nach der Erblindung überhaupt beobachtet und die ophthalmoskopische Diagnose auf Verschluss der Zentralarterie gestellt haben. Ich glaube aber, dass in diesen beiden Fällen, wie in meinem letzten Falle, die Diagnose auf vorhergegangenen Arterienverschluss eine, allerdings durch die ganzen Verhältnisse durchaus begründete, Vermutungsdiagnose war.

Um auf den in den obigen Notizen nur kurz skizzierten Befund noch etwas näher einzugehen, so geht aus der von Fräulein V. Steinbuch unter Kontrolle des Herrn Kollegen Fleischer angefertigten Abbildung (siehe Fig. 1 auf Taf. IX) deutlich hervor, dass feinste, stark geschlängelte Collateralen bestehen zwischen der Arteria nasalis und temporalis superior, sowie zwischen letzterer und dem Papillenrande und von hier aus, also indirekt, auch mit der Arteria temporalis inferior, die ihrerseits einen (wohl neugebildeten) Ast zu der kleinen Blutung zwischen Papille und Macula nach oben aussen sendet. Die Arteria nasalis superior steht nach unten hin in Verbindung mit der auf und ausserhalb der Papille stark verengten Vena nasalis inferior, und diese Collaterale zeigt wiederum eine Kommunikation mit einem am unteren Rande der Papille sich 
findenden Gefässschlingennetz, das auch einen feinen Ast aus der nahe an der Papille weiss eingescheideten Arteria nasalis inferior erhält. Von hier aus zur Arteria temporalis inferior ist eine Verbindung nicht mehr vorhanden. Es besteht also um die Papille ein fast vollkommener, aus feinen, stark geschlängelten neugebildeten Gefässen zusammengesetzter Gefässkranz, der alle vier erst am Papillenrande sichtbar werdenden Hauptäste der Zentralarterie miteinander verbindet und nur zwischen der Arteria temporalis inferior und dem Schlingennetze am unteren Papillenrande, gerade an der Austrittsstelle der stark gefüllten Vena temporalis inferior unterbrochen ist.

\section{Literaturübersicht.}

Über diese Frage der arteriellen Anastomosenbildung in der Netzhaut ist bisher in der Tat, wie Gonin (loc. cit. siehe oben) ganz richtig bemerkt, sehr wenig geschrieben worden, weil offenbar neugebildete Gefässverbindungen unter den Arterien der Netzhaut äusserst selten sind, während dasselbe Vorkommnis zwischen Venen oder Venen und Arterien der Netzhaut etwas häufiger zu sein scheint. Ich will kurz die bisherige Literatur besprechen.

Nettleship $(5,1891)$ beobachtete bei einem Manne (das Alter steht nicht angegeben) zwölf Monate nach Verschluss der Zentralarterie ein Netz von kleinen neugebildeten, stark geschlängelten Arterien um den rechten Optikus, welche die Netzhautzweige der Zentralarterie miteinander verbanden und auch teilweise aus dem Optikusgewebe selbst zu entspringen schienen. Dabei waren die Arterienzweige auf der Papille kaum sichtbar und in dünne blutleere Stränge verwandelt.

Einen ganz ähnlichen Fall beschrieb Gonin(13) im Jahre 1905. Auch hier handelte es sich bei einer 64jährigen Frau um eine plötzliche Erblindung des linken Auges, das drei Monate später untersucht wurde und folgenden Befund bot: Das Auge war amaurotisch. Die Papille war vollkommen atrophisch, alle Gefässe sehr eng. Die Retinalvenen waren ungefähr auf die Hälfte ihrer normalen Breite reduziert, die Arterien, die i. u. B. auf der Papille gar nicht zu sehen waren, schienen erst etwas entfernt rom Optikus in der Retina zu beginnen. Im a. B. konnte man jedoch erkennen, dass die Arterien als feine Füden auf der Papille begannen, und dass auf der temporalen Seite die Arterienäste durch vielfache feine Anastomosen miteinander in Verbindung standen. Die netzförmig geschlängelten Ver- 
bindungsarterien fasst der Autor als sekundär erweiterte präexistente Bahnen auf, also nicht als neugebildete Anastomosen.

Erst in letzter Zeit, im Juli 1913, publizierte Coats (18) eine analoge Beobachtung bei einem 27jährigen Manne, der nach verschiedenen Anfällen von Rheumatismus (zuletzt vor 2 Jahren) ein systolisches Geräusch an der Mitralis aufwies. Er hatte eines Morgens beim Erwachen eine Erblindung seines rechten Auges bemerkt, nachdem ihm tags zuvor ein Stück Lehm in dieses Auge gepflogen war, was zunächst keine Sehstörung zur Folge gehabt hatte. Die zwei Tage später vorgenommene ophth. Untersuchung liess das typische Bild des Zentralarterienverschlusses (Netzhauttrübung, roter Fleck in der Forea) erkennen, mit Ausnahme eines kleinen dreieckigen Bezirks an der temporalen Seite der Papille, der von einer kleinen cilioretinalen Arterie versorgt war. Dabei waren die Arterien relativ gut gefüllt und pulsierten bei Druck auf den Bulbus. R. $S=$ Handbewegungen temporalwärts. L. $S={ }^{6} \mid 6$. - Später blieben die oberen Gefässe enger als die unteren und die Retinaltrübung hellte sich unten viel schneller auf als oben, wo sie fast 1 Monat bestehen blieb. Schon vor Ablauf des ersten Monats wurde am Rande des oberen inneren Papillenquadranten ein Netzwerk feiner gekräuselter anastomotischer Gefässe bemerkt, die einen oberen Ast der Art. nas. inf. mit der Art. nas. sup. verbanden. Ausserdem bestand (hinter der oberen Vene) ein einfaches Verbindungsgefäss zwischen der Art. nas. und temp. sup, die beide auf der Papille stark verengt und (ebenso wie die obere Vene) von weissen Linien eingescheidet waren, während sie oberhalb der anastomotischen Vereinigungsstelle sich bedeutend erweiterten. - Nach fast 14 Monaten, wo die oberen Gefässe noch weiss eingescheidet erschienen, "hatten sich die Anastomosen bedeutend an Zahl vermindert. Nur ein gewundenes Gefäss, welches die (obere und untere) nasalen Arterien miteinander verband, war noch an der Stelle des früheren Plexus sichtbar." Das Sehvermögen hatte sich nicht gebessert. - Coats ist der Meinung, dass es sich in seinem Falle um die Erweiterung schon vorher bestehender, aber nicht sichtbarer anastomotischer Bahnen zwischen den oberen und unteren nasalen Arterien handelt, die die ungleiche Cirkulation zwischen den beiden Gefässgebieten auszugleichen berufen waren und auf der temporalen Seite nur deshalb nicht zur Entwicklung gekommen seien, weil das Vorhandensein der cilioretinalen Arterie auf dieser Seite gewissermassen einen mit relativ guter Cirkulation versehenen Keil dazwischen geschoben habe. 
Diese drei auf einen Stammverschluss der Zentralarterie folgenden peripapillären arteriellen Anastomosenbildungen entsprechen, abgesehen von verschiedenen Einzelheiten, ganz meinem dritten Falle.

Einen meinen beiden ersten Beobachtungen ähnlichen Fall ${ }^{1}$ ) teilte Königshöfer (11) im Jahre 1899 mit und bemerkte ausdrücklich, dass er in der Literatur etwas Ähnliches nicht gefunden habe ${ }^{2}$ ). Der Patient, ein 29jähriger gesunder Mann, erschien vier Tage nach einer spontan aufgetretenen Sehstörung und bot ophth. die typischen Zeichen eines frischen, ausserhalb des Optikus liegenden Verschlusses der rechten Arteria temporalis inferior mit entsprechendem skotomähnlichen Gesichtsfelddefekt, der nicht bis in die Peripherie reichte und später allmählich noch kleiner wurde. Blutungen waren nirgends in der Netzhaut zu sehen. Später kirschroter Fleck in der Macula. Nach Rückgang der Trübung konnte man eine kurze Unterbrechung der Arteria temporalis inferior ungefähr $1 / 2$ P. D. entfernt vom Papillenrande wahrnehmen, und peripher von dieser Stelle bildete sich innerhalb von zwei Monaten (nach der ersten Sehstörung) je eine arterielle Gefässverbindung mit einem Aste der Arteria nasalis inferior und der Arteria macularis inferior aus. Dieser Befund war vier Monate später, also ein halbes Jahr nach der Sehstörung unverändert. Zugleich war der Gesichtsfeldausfall auf ein Minimum reduziert, und die zentrale Sehschärfe, die früher etwas $<1,0$ betragen hatte, wieder normal $(5 / 4)$. Auch Königshöfer(11) ist der Meinung, dass die collateralen Verbindungen zwischen der Arteria temporalis inferior und nasalis inferior von Anfang an bestanden und später nur erweitert wurden, was er aus dem klinischen Befunde (rechtläufiger Blutstrom im peripheren Arterienstïcke von Anfang an, kein sektorenförmiger, sondern nur skotomähnlicher Ausfall, der die Peripherie nicht erreichte!) schliessen zu können glaubte.

In demselben Jahre 1899 berichtete Swan M. Burnett (9) über einen dem vorigen teilweise analogen $\mathrm{Fall}^{3}$ ). Ein sonst gesunder 85jähriger Mann erblindete plötzlich auf einem Auge. Bei der ersten Untersuchung war ein Sektor des Gesichtsfeldes erhalten geblieben und der Augenspiegel zeigte die Arterien fadenförmig, eine ganz unterbrochen, die Venen etwas gestaut bis auf eine, die unterbrochenen Blutstrom zeigte. Zum Bilde der sog. Embolie fehlte jedoch das Ödem der Netzhaut und der kirschrote Fleck in der Fovea.

1) Dieser Fall wird bei Gonin (13) und Coats(18) nicht erwähnt.

2) Er hat also die Beobachtung Nettleships (5, 1891, siehe oben) übersehen.

3) Auch dieser Fall ist von Gonin (13) und Coats (18) nicht berücksichtigt worden. 
Allmählich im Verlaufe von Monaten hob sich die Sehschärfe wieder auf $5 / 18$, vom Gesichtsfelde blieb die untere Hälfte ziemlich erhalten und reichte nach oben hin bis zu $10^{\circ}$ vom Fixierpunkte. Die allmählich eintretenden interessanten Veränderungen am Hintergrunde werden vom Autor an der Hand von Skizzen eingehend beschrieben. Die Venen füllten sich verhältnismässig rasch wieder, aus einer erfolgte eine Blutung. Auch die Arterien füllten sich wieder, nur eine zeigte noch lange zwei Unterbrechungen; an diesen Stellen bildeten sich auch später Knäuel neuer Kapillaren; ebenso bildeten sich interessante Anastomosen an anderer Stelle, an welcher eine Arterie gänzlich verschwunden war. Der Optikus wurde weiss.

Das sind meines Wissens die fünf einzigen Beobachtungen in der Literatur, welche meinen drei Fällen an die Seite gestellt werden können. Ausserdem werden noch einige Fälle beschrieben, in denen es neben venösen Gefässverbindungen, die im Vordergrunde standen, auch zu arteriellen Anastomosen kam.

So fand Schleich $(3,1885)$ in seinem Falle von „A neurysma arterio-venosum" bei einem achtjährigen gesunden Knaben, dessen rechtes Auge bei äusserlich normalen Verhältnissen vollkommen amaurotisch war, die stark erweiterten und geschlängelten Gefässe (Arterien und Venen) aus der Papille unter Aneurysmenbildung medusenhauptähnlich hervortretend und sich besonders in die temporale Peripherie verbreitend. Neben vielfachen Übergängen zwischen den erweiterten Venen und Arterien trat auch eine anastomotische Verbindung zwischen der Arteria temporalis superior und der Arteria temporalis inferior hervor. Das Verbindungsstück war, ebenso wie die verbundenen Arterien selbst, stark geschlängelt und erweitert (bis zum Vierfachen der normalen Dicke). Der Fall wird vom Autor in bezug auf seine Entstehung als congenitale Anomalie gedeutet.

Ferner fand Seydel $(8,1898)$ in seinem unter demselben Titel "A neurysma arterio-venosum" veröffentlichten Falle (18 jähriger Mann mit $S=6 / 9$ auf dem befallenen linken Auge) eine Verbindung zwischen dem Hauptaste der Arteria temporalis superior und einem von diesem über der Macula nach aussen abgehenden kleineren Aste. Die Verbindung war aber keine direkte, sondern wurde durch ein zwischen der Arteria und Vena temporalis superior zustande gekommenes Gefässschlingennetz vermittelt. Die Affektion wird vom Autor ebenfalls als congenitale Anomalie aufgefasst.

Endlich finde ich noch unter den sicher auf ein schweres Trauma zurückzuführenden Veränderungen im Falle von Eversbusch $(10,1899)$ 
neben zahlreichen anastomotischen Verbindungen zwischen Arterien und Venen auch eine solche (zweifache) zwischen der Arteria temporalis inferior und einem horizontal verlaufenden macularen Aste, dessen Anfangsstück an der Papille anscheinend auf eine längere Strecke obliteriert und unsichtbar war.

Diese kurze Übersicht über alle einschlägigen Fälle, die ich in der Literatur finden konnte, mag zeigen, wie seltene Beobachtungen die oben beschriebenen drei Fälle darstellen.

Dass anastomotische Verbindungen unter den Netzhautvenen nicht zu den Seltenheiten gehören, wenn sie auch im Beobachtungsmateriale des Einzelnen nicht gerade häufig sind, ist ja längst bekannt und verweise ich in bezug auf diese Frage auf die sehr gründliche Arbeit von Hormuth(11) aus der Axenfeldschen Freiburger Klinik, wo sich auch eine genaue Literaturzusammenstellung bis zum Jahre 1903 findet. Einen hierher gehörigen Fall hatte ich im vorigen Jahre bei einem 56 jährigen Manne mit Herzleiden nach Gelenkrheumatismus zu beobachten Gelegenheit. Teh habe den Fall in einem Vortrage (17) kurz besprochen, die ausfithrlichere Publikation desselben geschah jedoch in der unter meiner Anleitung angefertigten Dissertation von Adolf Pietzcker (20), wo auch der Hintergrund (loc. cit. Fig. 3, Taf. I) abgebildet ist. In diesem Falle bestanden neben der venösen Anastomosenbildung noch in den Glaskörper vorragende, neugebildete Gefässschlingen, die zum Teil von Arterien ausgingen, sowie eine kleine kreissegmentförmige sog. präretinale Blutung unterhalb der Maculagegend.

Ferner will ich darauf hinweisen, dass Greenwood $(14,1905)$ einer "Endarteritis mit Neubildung von Gefässen", über deren Natur im Referat nichts Näheres angegeben wird, Erwähnung tut, und Lawson $(16,1910)$ eine Anastomose zwischen einer Netzhaut-und einer Aderhautarterie gesehen haben will.

Endlich möchte ich der Vollständigkeit wegen nicht unterlassen, noch auf die hochinteressante und bisher wohl einzig dastehende Beobachtung von Schrader(15) hinzuweisen, bei der es sich um ein doppelseitiges sehr kompiliziert angeordnetes peripapilläres Gefässchlingennetz handelte, das aber hinter dem normalen Retinalgefässysteme lag, mit dem es keine Verbindungen hatte und vom Autor als ein abnorm nach vorn disloziertes und erkranktes Ciliargefässystem angesehen wird. Der Patient war ein sonst gesunder Mann von 32 Jahren. Die ausführlichen Einzelheiten der Beschreibung sind nicht kurz wiederzugeben. 


\section{Sohlussbemerkungen.}

Sieht man von den eben erwähnten drei Fällen (Schleich, Seydel, Eversbusch) ab, in denen die arterielle Gefässneubildung gewissermassen als Nebenbefund neben ausgedehnten venösen Anastomosen gefunden wurde, so bleiben im ganzen nur noch acht Fälle (Nettleship, Burnett, Königshöfer, Gonin, Coats, eigene Fälle I bis III) von reiner arterieller Anastomosenbildung übrig, die bis jetzt beobachtet wurden. Bei sieben von diesen acht Fällen schwankte das Alter zwischen 25 und 85 Jahren und betrug im Durchschnitt ungefähr 46 Jahre. Es waren fünf Männer [im Alter von 27, 29, 47 und 85 Jahren, einmal (Nettleship) steht das Alter nicht angegeben] und drei Frauen (im Alter von 25, 47 und 64 Jahren) befallen. Die Affektion war immer einseitig und betraf in zwei Fällen (Frauen) die linke Seite, in fünf Fällen (eine Frau, vier Männer) die rechte Seite, in einem Falle (Mann) steht die Seite im Referat nicht angegeben (Burnett).

Als Allgemeinbefund wurde in drei Fällen (Coats, eigene Fälle I und II) ein Herzleiden (Klappenfehler) nach Endocarditis arthritica festgestellt, in einem Falle (eigener Fall III) bestand periphere und cerebrale Arteriosklerose (Hemiplegie) mit Herzaffektion (Dilatatio cordis und systol. Geräusch), in zwei Fällen (Burnett, Königshöfer) war der Patient gesund, und in zwei Fällen (Nettleship, Gonin) steht über den Allgemeinzustand nichts vermerkt.

Von den acht Fällen wurden vier $(=50 \%)$ in Deutschland [davon drei $(=37,5 \%)$ in Württemberg, einer $(=12,5 \%)$ in Schlesien], zwei $(=25 \%)$ in England und je einer $(=12,5 \%)$ in Nordamerika und der französischen Schweiz beobachtet.

Diese acht Fälle haben das Gemeinsame, dass die Neubildung arterieller Anastomosen immer im Anschluss an einen Verschluss der Zentralarterie sich entwickelte. In der Hälfte der Fälle (Nettleship, Gonin, Coats, eigener Fall IIT) ging der kranzförmig um die Papille auftretenden Gefässneubildung ein Verschluss des Stammes der Zentralarterie voraus, wobei die Arterienäste auf der Papille in drei Fällen vollkommen obliteriert zu sein und erst am Rande des Optikus aufzutreten schienen. In den andern vier Fällen (Burnett, Königshöfer, eigene Fälle I und II) handelte es sich um den Verschluss eines oder mehrerer $\ddot{A}$ ste, und zwar der Arteria temp. inf. (Königshöfer), der Arteria temp. inf. und einer von der Arteria temp. sup. abzweigenden Maculaarterie (eigener Fall I) der Arteria nasalis inf. (eigener Fall II) und der unteren Arterienzweige (Burnett) 
In drei von diesen vier Fällen mit Astverschluss ging das peripher von der Verschlussstelle liegende Stück des Gefässes eine oder mehrere anastomotische Verbindungen ein, und zwar bei der verschlossenen Arteria temp. inf. im Falle Königshöfers mit der Arteria nasalis inf. und mit einer Maculaarterie, im eigenen Falle I mit einer kleineren submakularen temporalen Arterie und mit dem zentralen Stücke vor der Verschlussstelle, bei der verschlossenen Arteria nasalis inf. mit der Arteria temp. inf. und der Vena temp. sup., bei der verschlossenen Maculaarterie mit einer andern Maculaarterie.

Nur in einem Falle (Coats) wurde eine teilweise Rückbildung bereits vorhandener arterieller Anastomoseu beobachtet.

Alle acht Fälle sind seit dem Jahre 1891, also im Laufe der letzten 22 Jahre, zur Publikation gekommen, so dass der in der Einleitung citierte, damals (1891) mit voller Begründung von Fischer (4, S. 114) ausgesprochene Satz hente keine Berechtigung mehr hat.

Über die verschiedenen Möglichkeiten, wie man sich das $\mathrm{Zu}$ standekommen dieser anastomotischen Verbindungen unter den Netzhautarterien denken könne, haben sich Gonin(13) und Coats(18) in sehr eingehender Weise geäussert. Sie sind der Meinung, dass die kollateralen Bahnen in ophthalmoskopisch nicht sichtbarer Weise vorher vorhanden seien und nach dem Gefässverschluss durch stärkere Erweiterung für den Beobachter erst in die Erscheinung und auch in vermehrte Funktion träten.

Ich bin, insbesondere auf Grund der Erfahrung bei meinen beiden ersten Beobachtungen, wie ich dort schon angedeutet habe, der Ansicht, dass diese Erklärung jedenfalls nicht für alle Fälle zutrifft, sondern dass in seltenen Fällen nach Arterienverschluss eine wirkliche Neubildung arterieller Anastomosen in der Netzhaut, für die mir allerdings eine ausreichende Erklärung fehlt, tatsächlich vorkommt.

\section{Literaturverzeichnis.}

1869. 1) Hock, Ein Fall von Embolie der Art. centr. retinae. Wien. med. Presse. 186. Nr. 44.

2) Pagenstecher, Ein Fall von Verletzung des Nervus opticus mit Zerreissung der zentralen Gefässe and der dadurch bewirkten Veränderungen des Augenhintergrundes. v. Graefe's Arch. f. Ophth. Bd. XV, 1. S. 228 .

1885. 3) Schleich, G., Kasuistische Mitteilungen seltener Retinalerkrankungen: I. Aneurysma arteriovenosum, aneurysma circumscriptum et varix (aneurysmatiens ?) retinae. Mitteilungen aus der ophthalmiatrischen Klinik in Tübingen. 1885. Bd. II, 2, 8. 203.

1891. 4) Fischer, Über die Embolie der Arteria centralis retinae. Leipzig, Veit \& Comp. 1891 . 
5) Nettleship, Unusuell Appearances in a case of retinal embolism about 30 hours after its occurence. Festschr. für v. Helm holtz. S. 7 (S. 8).

1896. 6) Alexander, C., Wiederherstellung der Funtion bei einer 6 Jahre alten Embolie der Arteria centralis retinae. Ber. über d. 26. Vers. d. ophth. Ges. zu Heidelberg. S. 258.

7) Culberton, Artère rétinienne anormale. Ann. d'ocul. T. CXV. p. 290.

1898. 8) Seydel, Ein Aneurysma arterio-venosum (Varix aneurysmaticus) der Netzhaut. Areh. f. Augenheilk. Bd. XXXVIII. S. 157.

1899. 9) Burnett, Swan M., A case of obstrueted retinal circulation, with a series of pictures showing the changes in the vascular system during its re-etablishment and the formation of new vessels in the retina. Ophth. Record. Vol. VIII. p. 601. Ref. Zentralbl, f. Augenheilk. Jg. XXIII. S. 454.

10) Eversbusch, Ein auch in anatomischer Hinsicht bemerkenswerter Fall von einseitiger traumatischer Thrombose der Netzhautvenen, verbunden mit Blutung im Zentralkanal des Glaskörpers. Klin. Monatsbl. f. Augenheilk. 37. Jahrg. S. 1.

11) Königshöf er, Seltene Fälle. I. Anastomosenbildungen zwischen zwei Netzhantarterien bei einem Falle von Embolie der Arteria centralis retinae. Ophth. Klin. III. Jahrg. S. 133 (S. 134).

1903. 12) Hormuth, Über Anastomosenbildung und deren prognostische Bedentung bei thrombosierenden Erkrankungen im Gebiete der Vena centralis retinae. Klin. Monatsbl. f. Augenheilk. 41. Jahrg. Beilageheft. (Festschr. f. Manz.) S. 255.

1905. 13) Gonin, Rétablissement de la circulation rétinienne par des anastomoses à la suite d'une obstruction de l'artère centrale. Ann. d'ocul. T. CXXXIII. p. 167.

14) Greenwood, Obstruction in the retinal arteries. Journ. of the Amer. med. Assoc. March. 14. Ref. N.-M.'s Jahresber. 1905. S. 635.

1906. 15) Schrader, Ein endarteriitisch erkranktes cilioretinales Gefässsystem im Zusammenhang mit dem Circulus arteriosus n. optici. Zeitschr. f. Augenheilk. Bd. XVI, Beilageheft. (Festschrift für H. Kuhnt.) S. 81.

1910. 16) Lawson, Unusual variety of macula haemorrhage, with anastomosis between the retinal and choroidal circulation. Transact. of the Ophth. Soc. of the united Kingd. Vol. XXX. Ophth. Review. p. 255.

1912. 17) Harms, Über Gefässneubildung in der Netzhaut. Demonstrationsvortrag auf der Frühjahrsvers. d Verein. Württ. Augenärzte, 13. Sitz. am 2. Juni 1912. Ref. Klin. Monatsbl. f. Augenheilk. 50. Jahrg. Bd. II. (Neue Folge Bd. XIV.) S. 105. 1912.

1913. 18) Coats, George, Visible Anastomoses on the Papilla after obstruetion of the central artery. The Royal London Ophthalmic Hosp. Rep. Vol. XIX. Part. L. p. 78.

19) Kober, Paul, Klinische Untersuchungen über den Verschluss der Netzhautzentralarterie. Inaug--Diss. Tübingen 1913, und Deutschmanns Beiträge zur Augenheilk. Heft 85. Bd. IX. S. 405.

20) Pietzcker, Adolf, Weitere klinisch-kasuistische Beiträge zum Krankheitsbilde der sogenannten präretinalen Blutung. Inaug.-Diss. Tübingen 1913. Leipzig, W. Engelmann 1913. 
1.

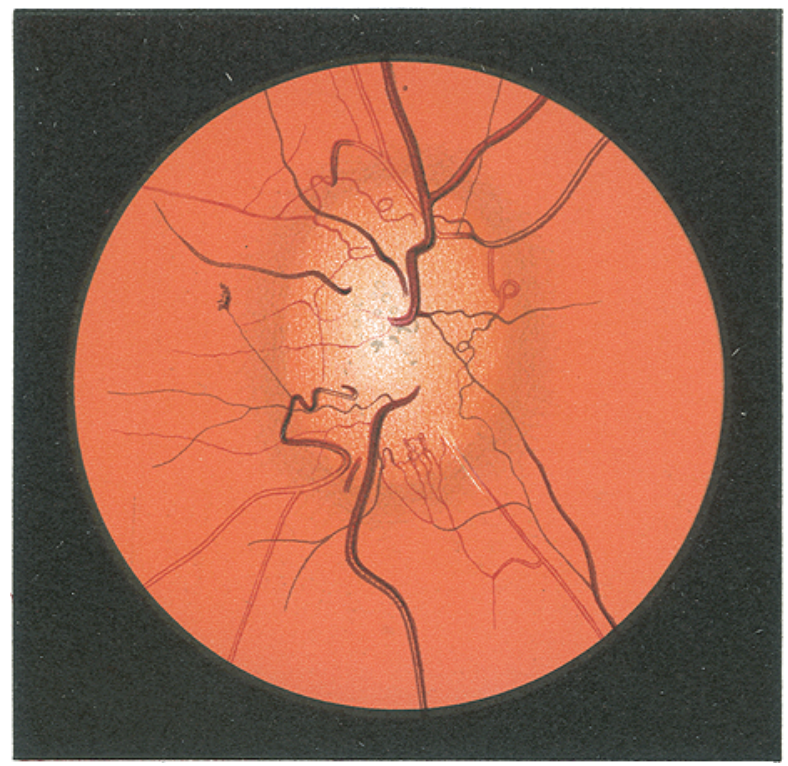

Rechtes Auge. Aufrechtes Bild.

Harms: Arterielle Anastomosenbildung in der Netzhaut.

2.

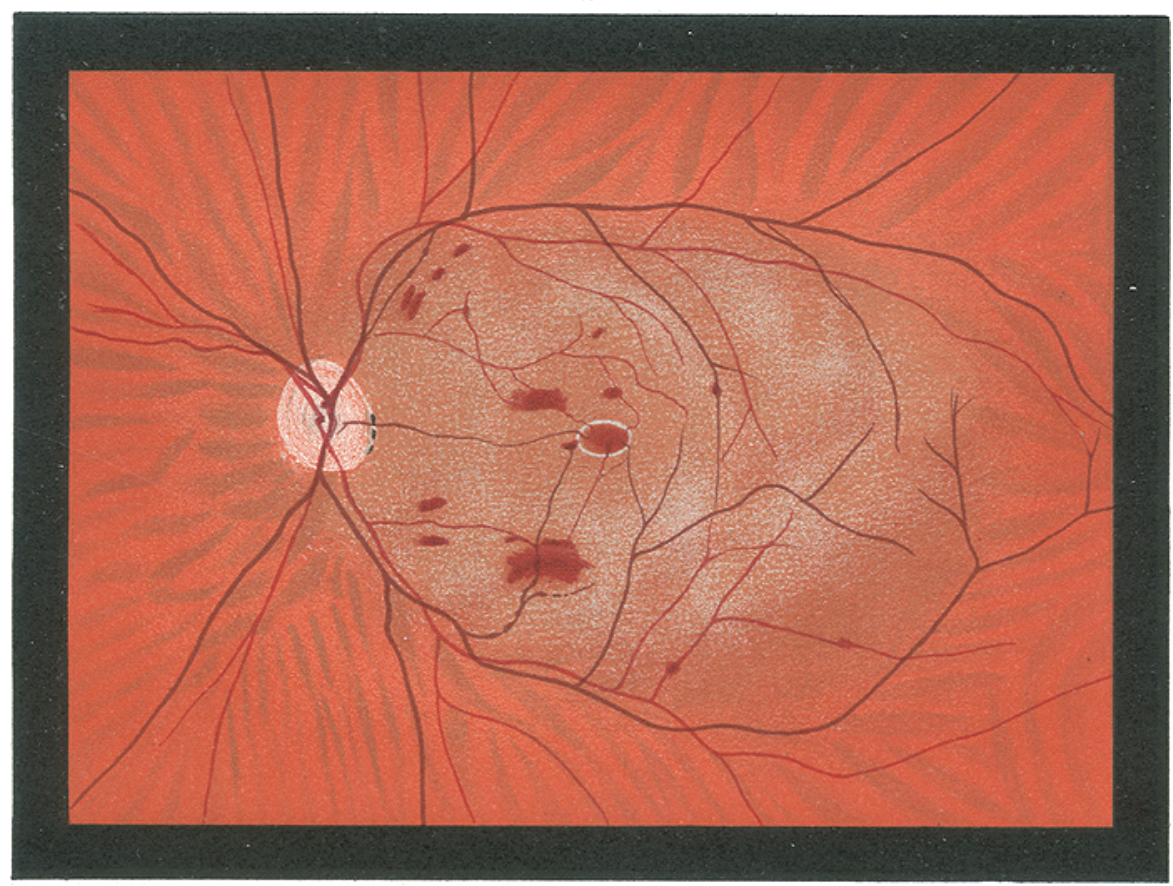

\section{Linkes Auge. Aufrechtes Bild.}

Harms: Aussergewöhntiche ophth. Befimde an der Macula lutea beim Stanmverschluss der Zentralarterie. (Fall IV: Dedematöse Ringfattenbildung um die Eovea)

Vilma Steinbuch al nat pinx.

It Anst ve Afmike Teiptig 